\title{
Recurrent Metastatic Digestive System Carcinoma
}

National Cancer Institute

\section{Source}

National Cancer Institute. Recurrent Metastatic Digestive System Carcinoma. NCI Thesaurus. Code C151905.

The reemergence of a metastatic digestive system carcinoma after a period of remission. 